\title{
Influence of Percutaneous Occlusion of Atrial Septal Defect on Left Atrial Function Evaluated Using 2D Speckle Tracking Echocardiography
}

\author{
Kazutaka Suzuki, ${ }^{1}$ MD, Taichi Kato, ${ }^{2}$ MD, Satoshi Koyama, ${ }^{1}$ MD, Tsutomu Shinohara, ${ }^{1}$ MD, \\ Sachiko Inukai, ${ }^{3} \mathrm{MD}$, Jun Sato, ${ }^{4} \mathrm{MD}$, Hidenori Yamamoto,${ }^{2} \mathrm{MD}$, Daisuke Omori, ${ }^{5} \mathrm{MD}$, \\ Shuichiro Yoshida, ${ }^{4} \mathrm{MD}$, Sho Takeda,${ }^{4} \mathrm{MD}$, Hiroshi Nishikawa, ${ }^{4} \mathrm{MD}$, \\ Naoki Ohashi, ${ }^{4}$ MD, Hajime Sakurai, ${ }^{6}$ MD and Shinji Saitoh, ${ }^{1}$ MD
}

\begin{abstract}
Summary
Percutaneous occlusion of atrial septal defect (ASD) has recently become a standard therapeutic strategy, but little is known about left atrial (LA) function thereafter. The present study aimed to determine LA function in 43 children with ASD and 13 controls based on LA strain measured by two-dimensional echocardiographic speckle tracking (2DE-ST). Among these children, 12 underwent surgery (ASD-S), 31 had device closure (ASD-D), and 13 were included as controls. LA strain was significantly decreased after ASD-D but was not significantly altered after ASD-S, indicating that percutaneous occlusion of an ASD might decrease LA function. Furthermore, the size of the ASD device negatively correlated with LA strain. These results imply that ASD occlusion devices negatively influence LA function and might be important when decided therapeutic strategies for ASD. LA strain measured by 2DE-ST should become a good indicator of LA function after ASD treatment in children.
\end{abstract}

(Int Heart J 2020; 61: 83-88)

Key words: Strain, Left atrial reservoir function, Left atrial conduit function, Left atrial booster pump function

A trial septal defect (ASD) with a left-to-right shunt is the most prevalent congenital heart disease. $\mathrm{Pa}$ tients with ASD develop right heart failure, atrial arrhythmias, and eventually pulmonary hypertension if a significant right heart volume load persists. ${ }^{1)}$ Since the risk of developing atrial fibrillation due to ASD without treatment increases with age, ASD should be treated before patients reach the age of 40 years. ${ }^{2)}$ Surgical outcomes have been very good for patients with ASD. Less invasive percutaneous occlusion using a specific device has recently been introduced as an alternative to surgery. The outcomes of percutaneous occlusion and surgery are comparable, and percutaneous occlusion has become more popular for ASD closure. ${ }^{3)}$

Although ventricular functions or volumes after percutaneous occlusion of ASD have been studied, ${ }^{4-7)}$ information about the effects of percutaneous occlusion on atrial performance is limited. ${ }^{8}$ Several methods have been developed to measure atrial function, ${ }^{9-13)}$ including left atrial (LA) strain, indicating LA reservoir, conduit, and booster pump function. ${ }^{14-16)}$ Representative methods of evaluating strain on echocardiographic Doppler images
(TDI) of tissues have the disadvantages of angle dependence and poor reproducibility and the potential for compromised evaluative accuracy. A method that is independent of angle is needed to accurately measure strain.

Two-dimensional echocardiographic speckle tracking (2DE-ST) is a novel, noninvasive way to resolve the issue of angle dependence. It systemically evaluates local or global myocardial movement, speed, and function by automatically tracking specific myocardial speckles. ${ }^{17}$ Atrial function has recently been analyzed using this method, ${ }^{18-21)}$ but it has not been applied to assessing LA function among patients with ASD.

The present study aimed to determine the effects of surgical or percutaneous occlusion of ASD on LA function in children using 2DE-ST.

\section{Methods}

Patients: We retrospectively investigated data from 59 children (28 males and 31 females, median age 8.0 years; IQR, 6.5-11.0 years) with ASD or Kawasaki disease who were admitted to Chukyo Hospital between April 2015

From the ${ }^{1}$ Department of Pediatrics and Neonatology, Nagoya City University Graduate School of Medical Sciences, Nagoya, Japan, ${ }^{2}$ Department of Pediatrics, Nagoya University Graduate School of Medicine, Nagoya, Japan, ${ }^{3}$ Department of Pediatrics, Nagoya Daini Red Cross Hospital, Nagoya, Japan, ${ }^{4}$ Department of Pediatric Cardiology, Japan Community Healthcare Organization Chukyo Children Heart Center, Nagoya, Japan, ${ }^{5}$ Department of Pediatrics, Anjo Kosei Hospital, Anjo, Japan and ${ }^{6}$ Cardiovascular Surgery, Japan Community Healthcare Organization Chukyo Children Heart Center, Nagoya, Japan.

Address for correspondence: Kazutaka Suzuki, MD, Department of Pediatrics and Neonatology, Nagoya City University Graduate School of Medical Sciences, Kawasumi 1, Mizuho-cho, Mizuho-ku, Nagoya 467-8601, Japan. E-mail: suzuki.k@med.nagoya-cu.ac.jp

Received for publication April 3, 2019. Revised and accepted October 1, 2019.

Released in advance online on J-STAGE January 17, 2020.

doi: 10.1536/ihj.19-173

All rights reserved by the International Heart Journal Association. 


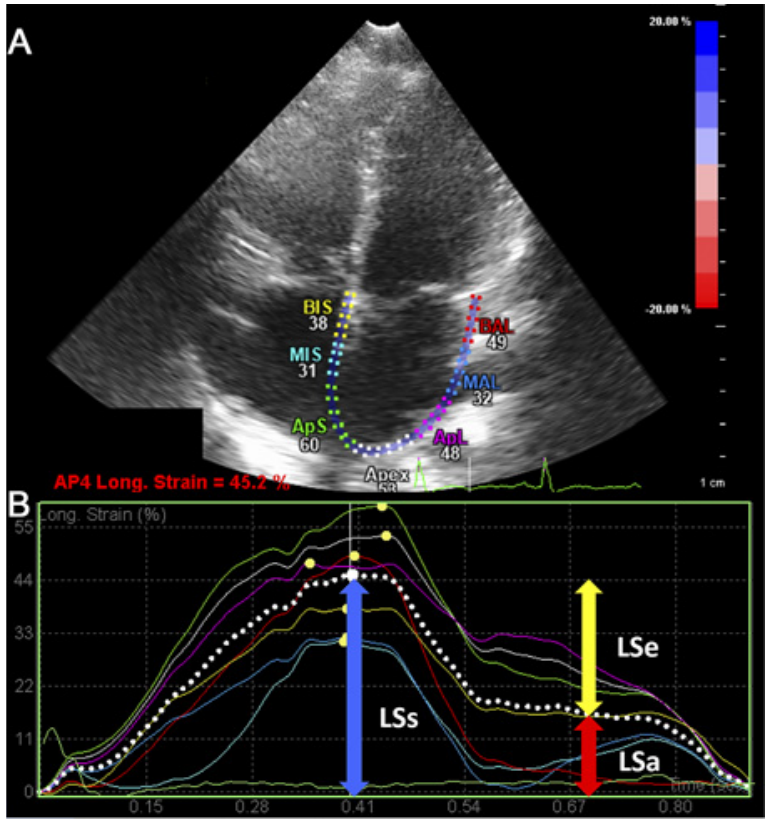

Figure. Longitudinal strain (LS) on LA determined using 2D speckle tracking analysis in apical four-chamber view. A: Representative four-chamber view with seven divided segments. B: LA strain at respective segments (the same color). White dotted line: mean value of LA strain. Blue, red, and yellow double-headed arrows: LSs, LSa, and LSe, respectively.

and August 2017. Three patients were excluded because of poor image quality. We therefore enrolled 56 patients in this study. Twelve had been treated by surgical repair of ASD (ASD-S), 31 had ASD device closure (ASD-D), and 13 with Kawasaki disease who were in the convalescence phase without coronary lesions served as controls. All had secundum ASD, and patients with abnormalities affecting hemodynamics such as complications requiring other treatments, pulmonary hypertension, atrioventricular valve regurgitation, and arrhythmia were excluded. All patients in the ASD-S group had undergone direct ASD closure. An AMPLATZER ${ }^{\mathrm{TM}}$ septal occluder (AGA Medical Corporation, Plymouth, MN, USA) was inserted into all patients in the ASD-D group. This group was subclassified according to the size of the ASD device (mm) inserted per body surface area $\left(\mathrm{BSA} ; \mathrm{m}^{2}\right)$ as small $(<15$ $\mathrm{mm} ; n=10)$, medium ( $\geq 15-20 \mathrm{~mm} ; n=10)$, or large ( $>$ $20 \mathrm{~mm} ; n=11)$.

Echocardiographic evaluation: One experienced cardiologist conducted all transthoracic echocardiographic assessments during the study using an IE-33 echo system (Philips North America LLC, Andover, MA, USA) before and after ASD treatment. Patients in the ASD-S group were examined one day before surgery and one day before discharge, and those in the ASD-D group were examined one day before and one day after intervention. Parasternal, apical, and subcostal images were acquired using a 5- or 8-MHz transducer.

We calculated ASD size and left ventricular ejection fraction from 2D echocardiography findings. Ratios of early ( $\mathrm{E}$ wave) to late (A wave) transmitral velocities (E/
A), left ventricular (LV)-TEI index, and pulmonary venous flow were obtained from pulse Doppler images. The velocity of LV inflow and outflow was recorded on pulse Doppler images. E' was measured at the septal and lateral mitral annulus velocities on images acquired in the apical four-chamber view. The ratio of mitral peak velocity of early filling (E) to early diastolic mitral annular velocity (E') was measured to estimate LV filling pressure. Pulmonary venous return velocities were measured from the apical four-chamber view by sampling the right upper pulmonary vein.

Measurement of LA strain using 2DE-ST: Two pediatric cardiologists who were blinded to demographic data measured LA strain by 2D speckle tracking using QLAB version 10.7 software (aDMQ; Philips North America) in apical four-chamber views of images that were acquired at a frame rate of $>50 \mathrm{~Hz}$. This software was originally devised to measure LV strain, but we applied it to measure LA strain as described. ${ }^{22)}$ Using this method, we evaluated speckle tracking in patients with ASD. ${ }^{23,24)}$ After one cardiac cycle was selected, we selected the septal and lateral corners of the mitral annulus and the LA roof in the systolic frame. The QLAB software automatically provided seven tracking regions on the LA wall and calculated the longitudinal strain (LS) using speckle tracking. Unsatisfactory tracking of the LA endocardium was manually adjusted. The LA walls were segmented into seven parts: the intra-atrial septum (IAS) of LA into three parts, the free wall (FW) of LA into three parts, and the roof of the LA into one part (Figure). We could not accurately calculate strain on the IAS due to the lack of a septal wall. Therefore, we calculated only LS of the FW as the average strain in three segments of the FW and did not include LS of IAS or global longitudinal strain. ${ }^{25}$ We calculated the average of the peak LS of FW during the systolic phase (LSs-FW) in three segments, which indicated LA reservoir function. LS of FW during the early diastolic phase (LSeFW) indicated LA conduit function. We calculated LS of FW during the late diastolic phase (LSa-FW), which indicates LA booster pump function, as the difference between LSs and LSe strain (Figure). ${ }^{14-16)}$

Statistical analysis: All data were statistically analyzed using the JMP software (SAS Institute, Cary, NC, USA). Data are presented as medians (IQR 25-75\%). Differences among ASD-S, ASD-D, and controls were assessed using nonparametric Kruskal-Wallis tests. Significant differences between two unpaired groups were assessed using nonparametric Steel-Dwass tests. Significant differences among the three groups classified by ASD device size were assessed using Kruskal-Wallis and Steel-Dwass tests. Correlations between ASD device size and LA strain were analyzed using Spearman's rank correlation test. We considered values with $P<0.05$ as being statistically significant. Inter- and intra-observer variability was assessed using Bland-Altman plots.

Informed consent: The Ethics Committee of the Nagoya City University Graduate School of Medical Sciences waived the need for informed consent due to the retrospective observational nature of the present study. The Ethics Committee at the Nagoya City University Graduate School of Medical Sciences and the Japan Community 
Table I. Demographic Data and Echocardiographic Parameters Before ASD Treatment

\begin{tabular}{|c|c|c|c|c|}
\hline & Control & ASD-D & ASD-S & $P$ \\
\hline$n$ & 13 & 31 & 12 & \\
\hline Age (months) & $102(73-132.5)$ & $95(81-130)$ & $107(70.5-153.8)$ & 0.9677 \\
\hline $\mathrm{BSA}\left(\mathrm{m}^{2}\right)$ & $0.87(0.6-1.1)$ & $0.9(0.8-1.15)$ & $0.9(0.75-1.22)$ & 0.5272 \\
\hline ASD size (mm) & & $12.9(10.5-16.7)$ & $19.2(16-25.1) *$ & 0.0011 \\
\hline $\operatorname{LVEF}(\%)$ & $72.2(66.2-76.5)$ & 76.4 (70.7-79.8) & $74.9(66.8-75.6)$ & 0.137 \\
\hline LVFS (\%) & $40.2(34.9-44.1)$ & $41.5(37.1-44.6)$ & $42.7(36.8-43.3)$ & 0.622 \\
\hline LV-TEI & $0.21(0.14-0.3)$ & $0.21(0.18-0.28)$ & $0.29(0.16-0.39)$ & 0.4254 \\
\hline MV-E (m/second) & $0.99(0.82-1.16)$ & $0.99(0.87-1.13)$ & $1.02(0.94-1.17)$ & 0.7157 \\
\hline MV-A (m/second) & $0.58(0.48-0.66)$ & $0.49(0.39-0.57)$ & $0.46(0.42-0.5)$ & 0.1586 \\
\hline $\mathrm{E} / \mathrm{A}$ & $1.68(1.5-1.93)$ & $2.14(1.81-2.64)$ & $2.14(1.48-2.62)$ & 0.0806 \\
\hline E/E' (sept) & $7.9(7-8.45)$ & $7.2(6.33-8.5)$ & $8(5.9-8.5)$ & 0.6583 \\
\hline E/E' (lat) & $5.8(4.55-6.6)$ & $4.8(4.1-5.7)$ & $5.65(5.28-7.15)$ & 0.1494 \\
\hline PVF (S/D) & $0.87(0.73-1.01)$ & $1.09(0.87-1.3)$ & $1.07(0.89-1.12)$ & 0.0944 \\
\hline PVF (A) (m/second) & $0.19(0.17-0.26)$ & $0.25(0.23-0.28)$ & $0.27(0.21-0.29)$ & 0.1386 \\
\hline LSs-FW (\%) & $38(34.5-49.5)$ & $43(34-46)$ & $37.5(30.5-41.8)$ & 0.618 \\
\hline LSe-FW (\%) & $30.1(26-37.4)$ & $32.3(24.6-39.6)$ & $27.5(23.7-35.6)$ & 0.6567 \\
\hline LSa-FW (\%) & $8.3(5.4-13.6)$ & $6(4-11.15)$ & $9.4(5.6-12.2)$ & 0.5874 \\
\hline
\end{tabular}

Data are expressed as medians and interquartile ranges (IQR). ASD indicates atrial septum defect; BSA, body surface area; FW, free wall; LS, longitudinal strain; LVEF, left ventricular ejection fraction; LVFS, left ventricular fractional shortening; MV-A, trans-mitral velocity of late wave; MV-E, trans-mitral velocity of early wave; and PVF, pulmonary venous flow. *Significant difference between ASD-D and ASD-S.

Table II. Echocardiographic Parameters After ASD Treatment

\begin{tabular}{|c|c|c|c|c|}
\hline & Control & ASD-D & ASD-S & $P$ \\
\hline$n$ & 13 & 31 & 12 & \\
\hline Age (months) & $102(73-132.5)$ & $95(81-130)$ & $107(70.5-153.8)$ & 0.9677 \\
\hline $\mathrm{BSA}\left(\mathrm{m}^{2}\right)$ & $0.87(0.6-1.1)$ & $0.9(0.8-1.15)$ & $0.9(0.75-1.22)$ & 0.5272 \\
\hline ASD size $(\mathrm{mm})$ & & $12.9(10.5-16.7)$ & $19.2(16-25.1) *$ & 0.0011 \\
\hline $\operatorname{LVEF}(\%)$ & $72.2(66.2-76.5)$ & $76.6(73-82.4)^{\dagger}$ & $77.4(73.7-81.6)$ & 0.0342 \\
\hline LVFS (\%) & $40.2(34.9-44.1)$ & $43.6(40-48.1)^{\dagger}$ & $43.8(41.2-47.7)$ & 0.0354 \\
\hline LV-TEI & $0.21(0.14-0.3)$ & $0.23(0.15-0.31)$ & $0.21(0.16-0.31)$ & 0.9857 \\
\hline MV-E (m/second) & $0.99(0.82-1.16)$ & $1.06(0.9-1.2)$ & $1(0.79-1.22)$ & 0.5528 \\
\hline MV-A (m/second) & $0.58(0.48-0.66)$ & $0.48(0.39-0.61)$ & $0.47(0.42-0.6)$ & 0.2299 \\
\hline $\mathrm{E} / \mathrm{A}$ & $1.68(1.5-1.93)$ & $2.02(1.66-2.73)$ & $2.12(1.58-2.56)$ & 0.0979 \\
\hline E/E' (sept) & $7.9(7-8.45)$ & $9.5(7.85-11.2)$ & $9.4(6.6-11.1)$ & 0.0583 \\
\hline E/E' (lat) & $5.8(4.55-6.6)$ & $5.9(4.85-7.25)$ & $6.2(5.5-8.2)$ & 0.3642 \\
\hline PVF (S/D) & $0.87(0.73-1.01)$ & $0.81(0.73-0.98)$ & $0.74(0.65-0.81)$ & 0.1802 \\
\hline $\operatorname{PVF}(\mathrm{A})(\mathrm{m} / \mathrm{second})$ & $0.19(0.17-0.26)$ & $0.24(0.21-0.29)$ & $0.21(0.17-0.26)$ & 0.2262 \\
\hline LSs-FW (\%) & $38(34.5-49.5)$ & $28(19.8-31) * \dagger$ & $36(27.3-42.5)$ & 0.0053 \\
\hline LSe-FW (\%) & $30.1(26-37.4)$ & $21.7(17.5-27.6)^{\dagger}$ & $26(20.2-29.7)$ & 0.0041 \\
\hline LSa-FW (\%) & $8.3(5.4-13.6)$ & $5.2(1.9-7.7) * \dagger$ & $8(6.4-15)$ & 0.0019 \\
\hline
\end{tabular}

Data are expressed as medians and interquartile ranges. ASD indicates atrial septum defect; BSA, body surface area; FW, free wall; LS, longitudinal strain; LVEF, left ventricular ejection fraction; LVFS, left ventricular fractional shortening; MV-A, trans-mitral velocity of late wave; MV-E, trans-mitral velocity of early wave; PVF, pulmonary venous flow; and sept, septal. Significant differences between *ASD-D and ASD-S, ${ }^{\dagger}$ ASD-D and control, and ${ }^{\ddagger} \mathrm{ASD}-\mathrm{S}$ and control.

Healthcare Organization Chukyo Children's Heart Center approved the study (Approval Number: 67-17-0035).

\section{Results}

Baseline characteristics of patients, outcomes, and echocardiographic parameters: Table I shows the demographic data and echocardiographic parameters before undergoing treatment for ASD. The ASD was closed without post-procedural complications in all patients in the device closure (ASD-D) and surgery (ASD-S) groups. Antiplatelet drugs were given to the ASD-D group for six months, and some patients in the ASD-S group received low-dose diuretics. Echocardiographic findings showed that LV contraction and dilation did not significantly differ among the ASD-S, ASD-D, and control groups. Atrial function assessed using 2DE-ST also did not significantly differ. The ASD was significantly larger in the ASD-S group than the ASD-D group because percutaneous occlusion was indicated for patients with an intact atrial septum margin and a relatively small ASD.

LA function after ASD treatment: Table II shows the echocardiographic findings after ASD treatment. Speckle tracking showed that LS of the FW significantly decreased 
Table III. Comparison of Left Atrial Strain Before and After Procedures in ASD-D Group

\begin{tabular}{lcccr}
\hline & Before procedure & After procedure & Mean difference 95\% CI & \multicolumn{1}{c}{$P$} \\
\hline LSs-FW (\%) & $43(34-46)$ & $28(19.8-31) *$ & $-14.43(-17.88$ to -10.99$)$ & $<0.0001$ \\
LSe-FW (\%) & $32.3(24.6-39.6)$ & $21.7(17.5-27.6) *$ & $-11.43(-14.55$ to -8.30$)$ & $<0.0001$ \\
LSa-FW (\%) & $6(4-11.15)$ & $5.2(1.9-7.7) *$ & $-3.54(-5.63$ to -1.44$)$ & 0.0020 \\
\hline
\end{tabular}

Data are expressed as medians and interquartile ranges. CI indicates confidence interval; FW, free wall; and LS, longitudinal strain. *Significant difference between before and after procedure.

Table IV. Comparison of Echocardiographic Parameters Among Small, Medium, and Large Devices

\begin{tabular}{lcccc}
\hline & Small & Medium & Large & $P$ \\
\hline$n$ & 10 & 10 & 11 & \\
Device/BSA & $9.42(8.95-13.66)$ & $18.5(17-19.16)$ & $23.1(20.6-25.3)$ & $<0.0001$ \\
Before procedure & & & & \\
$\quad$ LSs-FW (\%) & $43(35.5-49.5)$ & $36(33-44.5)$ & $40(28-46)$ & 0.3443 \\
LSe-FW (\%) & $35.7(24.8-42.2)$ & $30.6(26.1-37.3)$ & $29.7(22.6-41.5)$ & 0.6771 \\
$\quad$ LSa-FW (\%) & $9.7(4.45-14.2)$ & $5.3(2.6-14.3)$ & $5.2(3.3-10.8)$ & 0.4346 \\
After procedure & & & & \\
$\quad$ LSs-FW (\%) & $31.5(27.3-36.8)$ & $28(20.5-30.5)$ & $20(19-28) *$ & 0.0095 \\
LSe-FW (\%) & $28.1(22.3-31)$ & $23.3(19.1-27.7)$ & $17.9(16.1-19.1) *$ & 0.0047 \\
LSa-FW (\%) & $5.2(2.7-8)$ & $5.9(0.3-7.9)$ & $2.9(1.7-7.7)$ & 0.9288 \\
\hline
\end{tabular}

Data are expressed as medians and interquartile ranges. FW indicates free wall; and LS, longitudinal strain. *Significant difference between small and large devices.

Table V. Correlation Between ASD Device Size and LA Strain

\begin{tabular}{ccccc}
\hline & \multicolumn{2}{c}{ Before procedure } & \multicolumn{2}{c}{ After procedure } \\
\hline Parameter & $\rho$ & $P$ & $\rho$ & $P$ \\
LSs-FW & -0.2465 & 0.1892 & -0.5176 & 0.0034 \\
LSe-FW & -0.1502 & 0.4283 & -0.5809 & 0.0012 \\
LSa-FW & -0.1878 & 0.3203 & -0.0047 & 0.9812 \\
\hline
\end{tabular}

FW indicates free wall; and LS, longitudinal strain.

in the ASD-D group. Mean pre- and post-procedural differences in strain were significantly high in the ASD-D group (Table III).

The ASD-D group was subclassified depending on having a small, medium, or large device. We compared the strain among the three groups before and after treatment. LS of the systolic phase (LSs) and LS during the early diastolic phase (LSe) of FW significantly decreased with a large device, compared with a small device after treatment (Table IV). The ASD device size negatively correlated with LSs and LSe of FW (Table V).

Intra-observer and inter-observer variability: Intraobserver and inter-observer variability of measurements was evaluated in randomly selected patients. The interclass correlation coefficients for inter- and intra-observer variability of LS of the FW were 0.88 and 0.89 , respectively. The mean differences in inter- and intra-observer variability of LS of the FW were 0.229 (95\% confidence interval $[\mathrm{CI}],-1.00$ to 1.47$)$ and $-0.155(95 \% \mathrm{CI},-1.35$ to $1.04)$, respectively.

\section{Discussion}

We used speckle tracking to assess LA dysfunction that was not evident after percutaneous occlusion of an ASD. We found negative correlations between the size of ASD devices and LA strain. To our knowledge, this is the first study to use novel speckle tracking to compare the effects of catheter and surgical approaches on LA function in children with ASD and evaluate the correlation between device size and LA function.

We found that LSs, LSe, and LSa of FW were significantly decreased after ASD occlusion in the ASD-D group. A decrease in LSs of FW indicated an impaired atrium reservoir function in the ASD-D compared with ASD-S and control groups. The atrium reservoir function during the LV systolic phase was compromised by the device causing an extended disturbance, which led to a decrease in LA compliance. Since LV contraction and dilation were preserved, decreased LA reservoir function was affected by LA compliance rather than LV function. ${ }^{26)}$ Decreased LSe of FW reflects impaired the atrium conduit function. The atrium conduit function during the early diastolic LV filling phase is compromised due to resistance to the device. Decreases in LSa of FW reflect the impaired function of the atrial booster pump that becomes compromised by disturbed contraction caused by the device, which results in decreased LA contractility. ${ }^{26)}$ A previous study reported that reduced atrial function is a risk factor for the development of atrial fibrillation. ${ }^{27-29)}$ After ASD treatment, decreased LA strain might cause atrial arrhythmia, which would require long-term follow-up. Giovanni, et al. found that LA strain determined using TDI was significantly decreased in the ASD-S group compared 
with the control and ASD-D groups. ${ }^{8)}$ This might have been due in part to the angle dependence and poor reproducibility of TDI. Another report has suggested that color Doppler myocardial imaging cannot significantly discriminate an ASD device from the normal atrial wall. Color Doppler myocardial imaging is not appropriate for accurate evaluation of LA function. ${ }^{11)}$

Regional strain was also significantly decreased after ASD closure in the ASD-D group. Insertion of the ASD device reduced the LS of FW, whereas FW was not directly obstructed by the ASD device. Anatomically, the atrial walls consist of circumferential and longitudinal muscular bundles. ${ }^{23)}$ Therefore, the large device used to close the ASD might have initially disrupted these bundles in the IAS directly, followed by the subsequent disruption of the free wall of the left atrium. ${ }^{30)}$ Furthermore, LSs-FW and LSe-FW were decreased more by a larger device in the ASD-D group. In fact, the size of the ASD device negatively correlated with the LA reservoir and conduit function. These data suggest that careful follow-up of LA function might be needed for patients with larger devices. A previous study found no significant correlation between device size and atrial function between a patent foramen ovale and ASD. ${ }^{8)}$ Our results differed from these, in part, because we measured the atrium strain using 2DE-ST, whereas the previous study measured using TDI.

We confirmed that the traditional parameters of atrial function, transmitral velocities of late wave (MV-A), ratios of systolic to diastolic pulmonary venous flow (PVF), and PVF of atrial reversal flow did not significantly decrease after ASD closure in both the ASD-D and ASD-S groups. ${ }^{931,32)}$ The evaluation of mitral Doppler flow and mitral annular motion suggested that atrial stiffness increased after device placement. ${ }^{12,33)}$ However, these parameters did not directly reflect atrial functions. Contrary to these traditional parameters, our results showed that strain determined by speckle tracking could identify small changes in LA dysfunction.

This study had some limitations. Firstly, twodimensional analysis of atrial strain from the apical fourchamber view might not have assessed the function of the entire atrium. Regardless of LA morphology, the assessment of LA function was too difficult, and a 3DE method of assessing LA strain has not been established. Secondly, we evaluated a short-term endpoint after ASD treatment, so an accurate long-term prognosis in this cohort cannot be elucidated by this study design. However, a previous study that evaluated LA strain immediately and six months after ASD closure found no difference. ${ }^{23)}$ Although validation is needed, LA performance in the short and long terms might correlate.

In conclusion, analyzing LA strain using $2 \mathrm{DE}$ speckle tracking allowed simple and valuable assessment of the LA reservoir, conduit, and booster pump function of ASD after treatment. We showed that LA function was decreased after ASD occlusion in the ASD-D group compared with the ASD-S group. Furthermore, LA reservoir and conduit function negatively correlated with device size. These findings warrant further investigation of the relationship between long-term atrial function and complications after ASD treatment, which would facilitate more appropriate decisions regarding treatment strategies.

\section{Acknowledgments}

We thank Satoshi Osaga for assistance with statistical processing.

\section{Disclosure}

Conflicts of interest: The authors have no conflicts of interest to declare.

\section{References}

1. Oliver JM, Gallego P, González A, Benito F, Mesa JM, Sobrino JA. Predisposing conditions for atrial fibrillation in atrial septal defect with and without operative closure. Am J Cardiol 2002; 89: $39-43$.

2. Gatzoulis MA, Freeman MA, Siu SC, Webb GD, Harris L. Atrial arrhythmia after surgical closure of atrial septal defects in adults. N Engl J Med 1999; 340: 839-46.

3. Du ZD, Hijazi ZM, Kleinman CS, Silverman NH, Larntz K, Amplatzer Investigators. Comparison between transcatheter and surgical closure of secundum atrial septal defect in children and adults: results of a multicenter nonrandomized trial. J Am Coll Cardiol 2002; 39: 1836-44.

4. Castaldi B, Santoro G, Di Salvo G, et al. Impact of the Amplatzer atrial septal occluder device on left ventricular function in pediatric patients. Pediatr Cardiol 2013; 34: 1645-51.

5. Samiei N, Bayat F, Moradi M, et al. Comparison of the response of the right ventricle with endovascular occlusion and surgical closure in adults with atrial septal defect one year after intervention. Clin Med Insights Cardiol 2010; 4: 143-7.

6. Di Salvo G, Drago M, Pacileo G, et al. Comparison of strain rate imaging for quantitative evaluation of regional left and right ventricular function after surgical versus percutaneous closure of atrial septal defect. Am J Cardiol 2005; 96: 299-302.

7. Pawelec-Wojtalik M, Wojtalik M, Mrowczynski W, Surmacz R, Quereshi SA. Comparison of cardiac function in children after surgical and Amplatzer occluder closure of secundum atrial septal defects. Eur J Cardio Thorac Surg 2006; 29: 89-92.

8. Di Salvo G, Drago M, Pacileo G, et al. Atrial function after surgical and percutaneous closure of atrial septal defect: a strain rate imaging study. J Am Soc Echocardiogr 2005; 18: 930-3.

9. Yılmazer MM, Güven B, Vupa-Çilengiroğlu Ö, et al. Improvement in cardiac structure and functions early after transcatheter closure of secundum atrial septal defect in children and adolescents. Turk J Pediatr 2013; 55: 401-10.

10. Di Salvo G, Pacileo G, Castaldi B, et al. Two-dimensional strain and atrial function: a study on patients after percutaneous closure of atrial septal defect. Eur J Echocardiogr 2009; 10: 256-9.

11. Di Salvo G, Pacileo G, Caso P, et al. Strain rate imaging is a superior method for the assessment of regional myocardial function compared with Doppler tissue imaging: a study on patients with transcatheter device closure of atrial septal defect. J Am Soc Echocardiogr 2005; 18: 398-400.

12. Lange A, Coleman DM, Palka P, Burstow DJ, Wilkinson JL, Godman MJ. Effect of catheter device closure of atrial septal defect on diastolic mitral annular motion. Am J Cardiol 2003; 91: $104-8$

13. Aslan M, Erturk M, Turen S, et al. Effects of percutaneous closure of atrial septal defect on left atrial mechanical and conduction functions. Eur Heart J Cardiovasc Imaging 2014; 15: 111724.

14. Vianna-Pinton R, Moreno CA, Baxter CM, Lee KS, Tsang TS, Appleton CP. Two-dimensional speckle-tracking echocardiography of the left atrium: feasibility and regional contraction and 
relaxation differences in normal subjects. J Am Soc Echocardiogr 2009; 22: 299-305.

15. Arat N, Sökmen Y, Altay H, Ozcan F, Ilkay E. Left and right atrial myocardial deformation properties in patients with an atrial septal defect. Echocardiography 2008; 25: 401-7.

16. Sirbu C, Herbots L, D'Hooge J, et al. Feasibility of strain and strain rate imaging for the assessment of regional left atrial deformation: a study in normal subjects. Eur J Echocardiogr 2006; 7: 199-208.

17. Shi J, Xing Y, Qian J, et al. Early assessment of left ventricular function by layer-specific strain and its relationship to pulsatile arterial load in patients with coronary slow flow. Int Heart $\mathbf{J}$ 2019; 60: 586-92.

18. Kamijima R, Suzuki K, Izumo M, et al. Predictors of exerciseinduced pulmonary hypertension in patients with asymptomatic degenerative mitral regurgitation: mechanistic insights from 2D speckle-tracking echocardiography. Sci Rep 2017; 7: 40008.

19. Yuda S, Muranaka A, Miura T. Clinical implications of left atrial function assessed by speckle tracking echocardiography. J Echocardiogr 2016; 14: 104-12.

20. Sakata K, Uesugi Y, Isaka A, et al. Evaluation of right atrial function using right atrial speckle tracking analysis in patients with pulmonary artery hypertension. J Echocardiogr 2016; 14: 30-8.

21. Hsu PC, Lee WH, Chu CY, et al. Prognostic role of left atrial strain and its combination index with transmitral E-wave velocity in patients with atrial fibrillation. Sci Rep 2016; 6: 17318.

22. Ancona R, Comenale Pinto S, Caso P, et al. Left atrium by echocardiography in clinical practice: from conventional methods to new echocardiographic techniques. Sci World J 2014; 2014: 451042.

23. Vitarelli A, Gaudio C, Mangieri E, et al. Bi-atrial function before and after percutaneous closure of atrial septum in patients with and without paroxysmal atrial fibrillation: A 2-D and 3-D speckle tracking echocardiographic study. Ultrasound Med Biol 2018; 44: 1198-211.

24. Vitarelli A, Mangieri E, Gaudio C, Tanzilli G, Miraldi F, Capo- tosto L. Right atrial function by speckle tracking echocardiography in atrial septal defect: prediction of atrial fibrillation. Clin Cardiol 2018; 41: 1341-7.

25. Hajizeinali A, Iri M, Hosseinsabet A. Assessment of the right and left atrial functions at midterm after surgical and device atrial septal defect closure: A 2-dimensional speckle-tracking echocardiographic study. J Ultrasound Med 2019; 38: 1979-93.

26. Boyd AC, Cooper M, Thomas L. Segmental atrial function following percutaneous closure of atrial septum using occluder device. J Am Soc Echocardiogr 2009; 22: 508-16.

27. Sarvari SI, Haugaa KH, Stokke TM, et al. Strain echocardiographic assessment of left atrial function predicts recurrence of atrial fibrillation. Eur Heart J Cardiovasc Imaging 2016; 17: 660-7.

28. Tsai WC, Lee CH, Lin CC, et al. Association of left atrial strain and strain rate assessed by speckle tracking echocardiography with paroxysmal atrial fibrillation. Echocardiography 2009; 26: 1188-94.

29. Providência R, Faustino A, Ferreira MJ, et al. Evaluation of left atrial deformation to predict left atrial stasis in patients with non-valvular atrial fibrillation-a pilot-study. Cardiovasc Ultrasound 2013; 11: 44.

30. Wang K, Ho SY, Gibson DG, Anderson RH. Architecture of atrial musculature in humans. Br Heart J 1995; 73: 559-65.

31. Schroh AM, Laghezza LB, Domínguez PJ, et al. Pattern of pulmonary venous flow in patients with ostium secundum atrial septal defect. Eur J Echocardiogr 2009; 10: 244-9.

32. Lam YY, Fang F, Yip GW, Li ZA, Yang Y, Yu CM. New pulmonary vein Doppler echocardiographic index predicts significant interatrial shunting in secundum atrial septal defect. Int J Cardiol 2012; 160: 59-65.

33. Hanséus KC, Björkhem GE, Brodin LA, Pesonen E. Analysis of atrioventricular plane movements by Doppler tissue imaging and M-mode in children with atrial septal defects before and after surgical and device closure. Pediatr Cardiol 2002; 23: 152-9. 